\title{
Highlights from the LHC
}

\section{Geoffrey N Taylor ${ }^{1}$}

ARC Centre of Excellence for Particle Physics at the Terascale

The University of Melbourne,

Melbourne, Australia

E-mail: gntayloreunimelb.edu.au

In this talk, is presented an update of the status of operations of the LHC with emphasis on the ATLAS and CMS results and plans. Results from the 2011 running period are presented, including the status of the search for evidence of the Higgs boson. A sample of other physics results is provided to highlight the substantial progress that has already been made in the initial $7 \mathrm{TeV}$ running of the LHC. Indications from first running experience with $8 \mathrm{TeV}$ operation have raised expectations for a very successful year of data taking in 2012.

The 30 International Symposium on Lattice Field Theory

Cairns, Australia

June 24-29, 2012

$1 \quad$ Speaker 


\section{Introduction}

The Large Hadron Collider (LHC) at the CERN has been operating successfully since 2010. The data sets amassed by ATLAS and CMS (from integrated luminosities of $5.0 \mathrm{fb}-1$ in 2011 and $6.2 \mathrm{fb}-1$ of so far in 2012) are already impressive and powerful. This talk provides an overview of the results reported to date and gives an outlook of what is expected in the future.

\subsection{Goals of the LHC}

The key questions posed for the LHC are:

- What is the origin of the electroweak symmetry breaking and the origin of mass?

- How can the imbalance in the Universe's matter-antimatter be explained?

- Were the fundamental forces unified at an earlier epoch?

- What is the nature of dark matter?

- Are there extra dimensions of space-time.

Progress on Higgs searches, with the goal of answering the first of these will be described. Some examples of results aimed at the other topics will be presented.

It is expected that new particles will appear at the TeV scale in reach of the LHC. The terascale, defined by the $10^{12} \mathrm{eV}$ energy scale corresponds to the electroweak unification scale.

\subsection{Initial Operation}

The Large Hadron Collider (LHC) now in operation at CERN since late 2010, produced $5 \mathrm{fb}^{-1}$ in 2011 and has been operating flawlessly in 2012. Four major experiments are in operation at the Large Hadron Collider - ATLAS, CMS, LHCb and ALICE. ATLAS and CMS are studying electroweak symmetry breaking, searching for evidence of supersymmetry, extra dimensions, and for other exotics. The LHCb collaboration is making precision measurements of the CKM matrix and carrying out precision tests of the Standard Model. ALICE is studying the properties of the quark gluon plasma. Smaller specialist experiments, ALPHA and TOTEM, will make small angle scattering measurements for determining precise luminosity measurements.

2012 will see the completion of the first major data taking period for the LHC where Atlas and CMS are expecting in excess of $15 \mathrm{fb}^{-1}$ with $8 \mathrm{TeV}$ of proton collision energy. It is expected that such a dataset will provide a definitive answer on the existence or not of a low mass Higgs boson. By the time of the scheduled machine development technical stop in mid-June the LHC had delivered $6.65 \mathrm{fb}^{-1}$ with maximum instantaneous luminosity $7 \times 10^{33} \mathrm{~cm}^{-2} \mathrm{~s}^{-1}$. This is a remarkable achievement. With the increasing level asked the, and in particular with a $50 \mathrm{~ns}$ collision frequency of current operations, comes the difficulty of multiple interactions that bunch crossing. At maximum instantaneous luminosity, ATLAS and CMS are recording in excess of 20 primary vertices per beam crossing. 


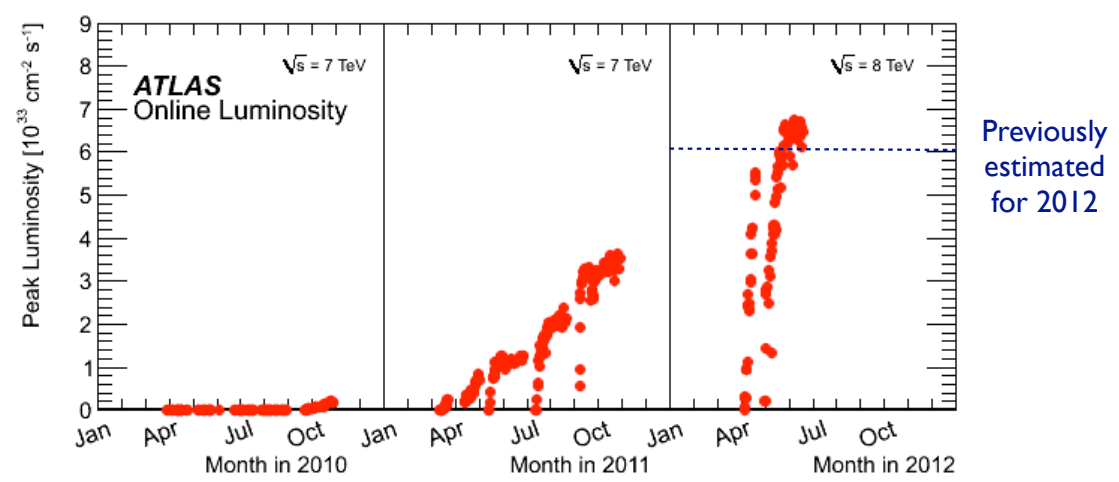

Figure 1. The peak instantaneous luminosity delivered to ATLAS per day versus time during the p-p runs of 2010, 2011 and early 2012. The online luminosity measurement is used for this plot [1].

The impressive operation of both the experiments and the LHC has resulted in accurate measurements of standard model processes. Rediscovering the standard model is an important test of the understanding of the experiments, their calibrations, and their resolution. The impressive accuracy of these results and their correlation with predicted values gives great confidence in the quality of the data and the analyses being produced by these collaborations so early in the operation of their experiments.

In 2011, with only $5 \mathrm{fb}^{-1}$ of data, ATLAS and CMS announced results on searches for the Higgs boson. Via key channels such as Higgs to $\gamma \gamma$ and Higgs to WW, a large range of possible Higgs masses have been eliminated.

\section{Re-discovering the Standard Model}

Not only does operation of the new machine at vastly higher energy and initial luminosity pose the challenge of understanding in the extraction of physics, but the major effort in understanding the operation of the enormously complicated detectors must be well advanced before any results can be seriously considered. This included the painstaking tasks of data recording and validation, trigger efficiency and bias correction, energy and momentum calibration and modelling, and physics extraction through detailed comparison with welldeveloped simulations and with known properties of high energy proton interactions and the plethora of processes that result. 

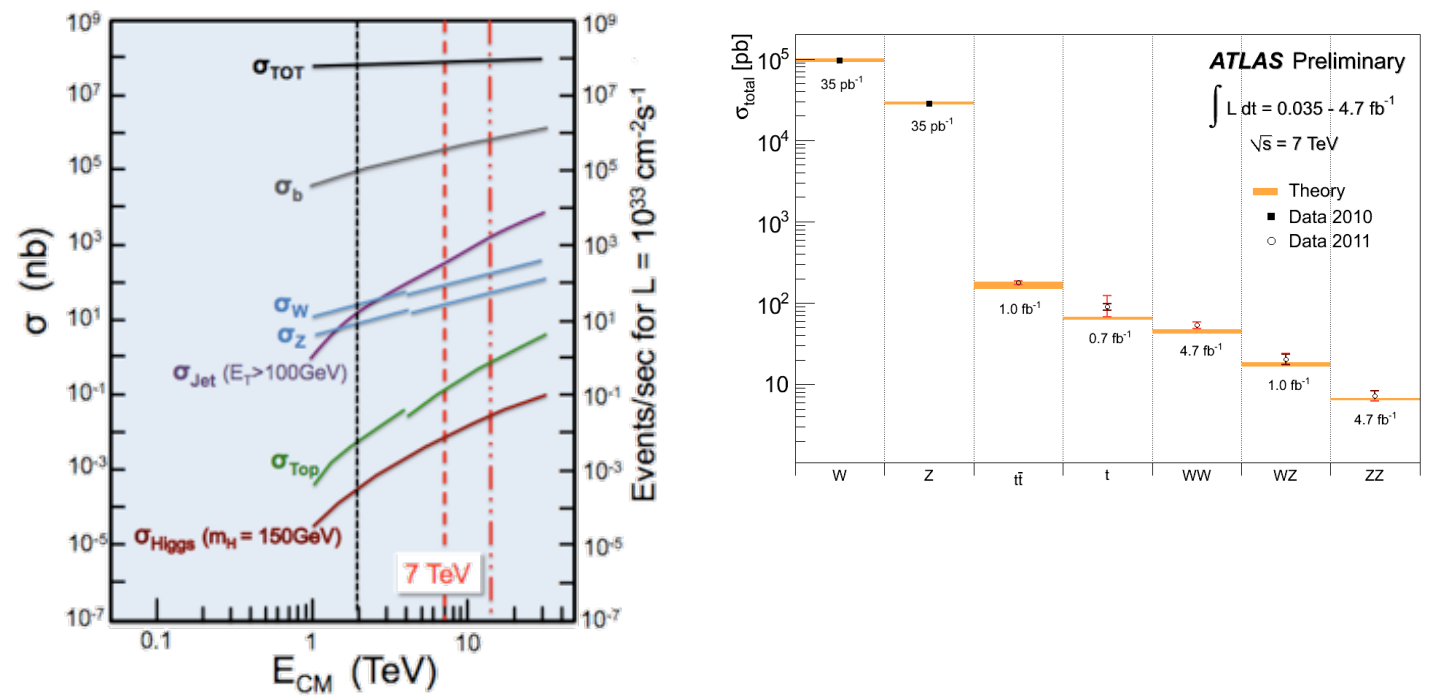

Figure 2. Expected cross-sections versus collision energy, and a compilation of measured and expected cross sections for various SM processes [4].

The process of comparison with known properties of particles and their interactions can be likened to a re-discovery of the Standard Model of particle physics. This is an essential first step in gaining confidence that the collaborations understand their detectors and in quantifying the resolution and capability of extracting signal from background. The SM processes become the background for new discoveries and will most often dominate the measurements. Precision understanding of these processes and the ability to understand their appearance in the detectors with a high level of understanding is thus the critical foundation upon which any claim for new physics will be based.

Figure 2 shows Atlas measurements of cross sections for a range of standard model processes, that cover nearly 5 orders of magnitude in cross section, and that make critical use of a variety of experimental techniques and sub-detectors. In all comparisons, these cross-sections show excellent comparison with expectation.

\section{Operational Conditions}

Figure 3(a) shows integrated luminosity delivered by the LHC and recorded by the CMS detector for the 2012 running until the first LHC machine development technical stop, in June. A very similar plot exists for the ATLAS experiment. The LHC has successfully delivered a "luminosity balanced" beam to $\mathrm{LHCb}$ to keep that experiment operating at its maximum trigger rate.

Figure 1 shows a plot of the peak luminosity achieved in the beam fills during this period. The extraordinary success in raising the luminosity through the first period of 2012 operations has resulted in the large delivered beam rate, achieving the goal of $6.6 \mathrm{fb}^{-1}$ integrated luminosity, 
even with the slower-than-expected beginning. This is testament to operations expertise, but also to the high degree of instrumentation and automation developed for the LHC.

With the experiments demanding maximum luminosity in order to be sensitive to new physics come increased experimental challenges. Multiple interactions per beam crossing is a key example. Whilst in 2011 the average number of interactions per beam crossing was in the range 6-12, depending upon the final focus conditions of the LHC, by June 2012 this has increased to 16 with values of 30 becoming commonplace (see figure $3(\mathrm{~b})$ ).
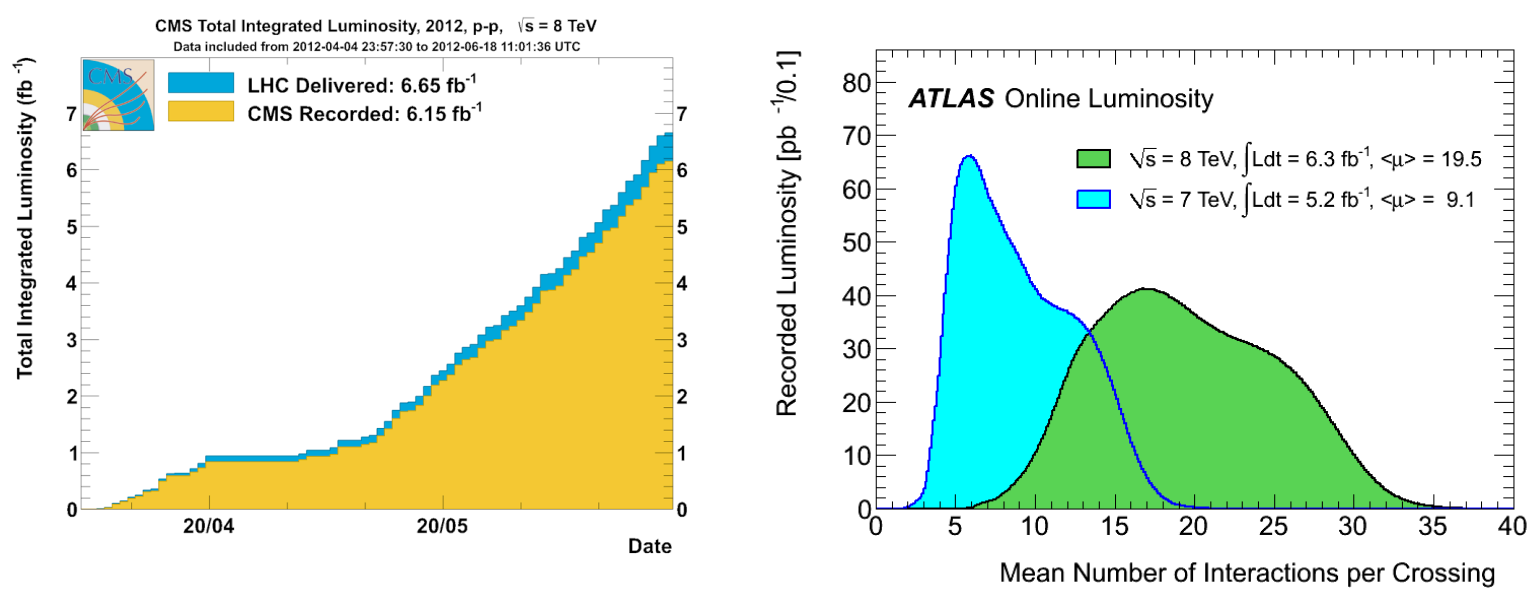

Figure 3 (a) The integrated luminosities [3] and (b) the luminosity-weighted distribution of the mean number of interactions per crossing, $\mu$, for the 2011 and 2012 (April 4th and June $18^{\text {th }}$ ) data. $\mu=\mathrm{L}_{\text {bunch }} \mathrm{x} \sigma_{\text {inel }} / \mathrm{f}_{\mathrm{r}}$ where $\mathrm{L}_{\text {bunch }}$ instantaneous luminosity, $\sigma_{\text {inel }}$ is the inelastic cross section (assumed $71.5 \mathrm{mb}$ at $7 \mathrm{TeV}$ and $73.0 \mathrm{mb}$ at $8 \mathrm{TeV}$ collision energy), $\mathrm{n}_{\text {bunch }}$ is the number of bunches and $\mathrm{f}_{\mathrm{r}}$ is the bunch revolution frequency [1]

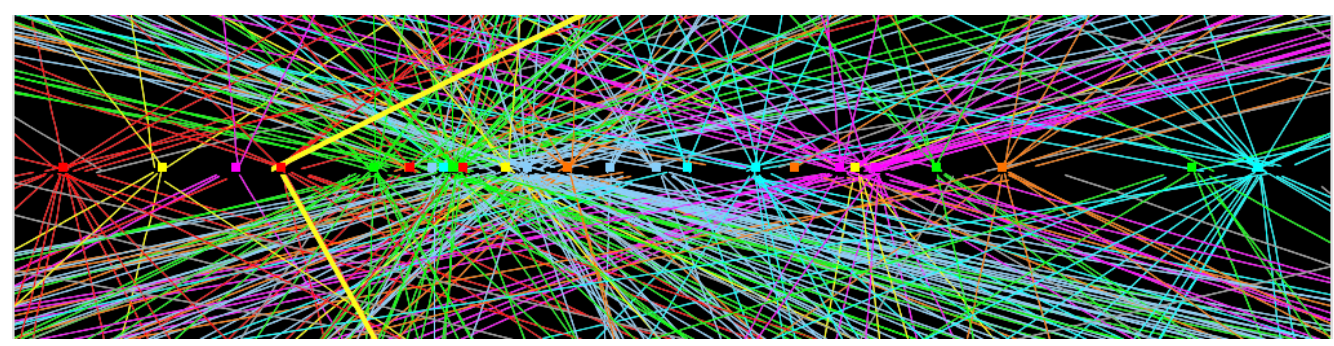

Figure 4 An example of a multi-interaction event from the ATLAS detector with tracks from the high-pT triggered interaction highlighted amongst the 24 other "pile-up" interactions.

\section{Status of Higgs Searches}

With the search for the Higgs boson driving the research program of ATLAS and CMS, initial results received great attention when presented at CERN in December, 2011. The two key channels were $\mathrm{H}$ to $\gamma$ and $\mathrm{H}$ to $\mathrm{WW}^{*}$. Figure 5 shows the results from CMS measured 2- 
photon invariant mass spectrum over the mass range of interest $110-150 \mathrm{GeV}$ with the extracted ratio $95 \%$ confidence limit (CL) for the measured cross section for $\gamma$ pairs, to the expected cross section from SM contributions to this final state in the absence of a Higgs boson [4]. Whilst being consistent with the SM predictions, a small but tantalising excess is seen near a diphoton mass of $125 \mathrm{GeV}$. Interestingly, a small excess is also seen in ATLAS data [5].

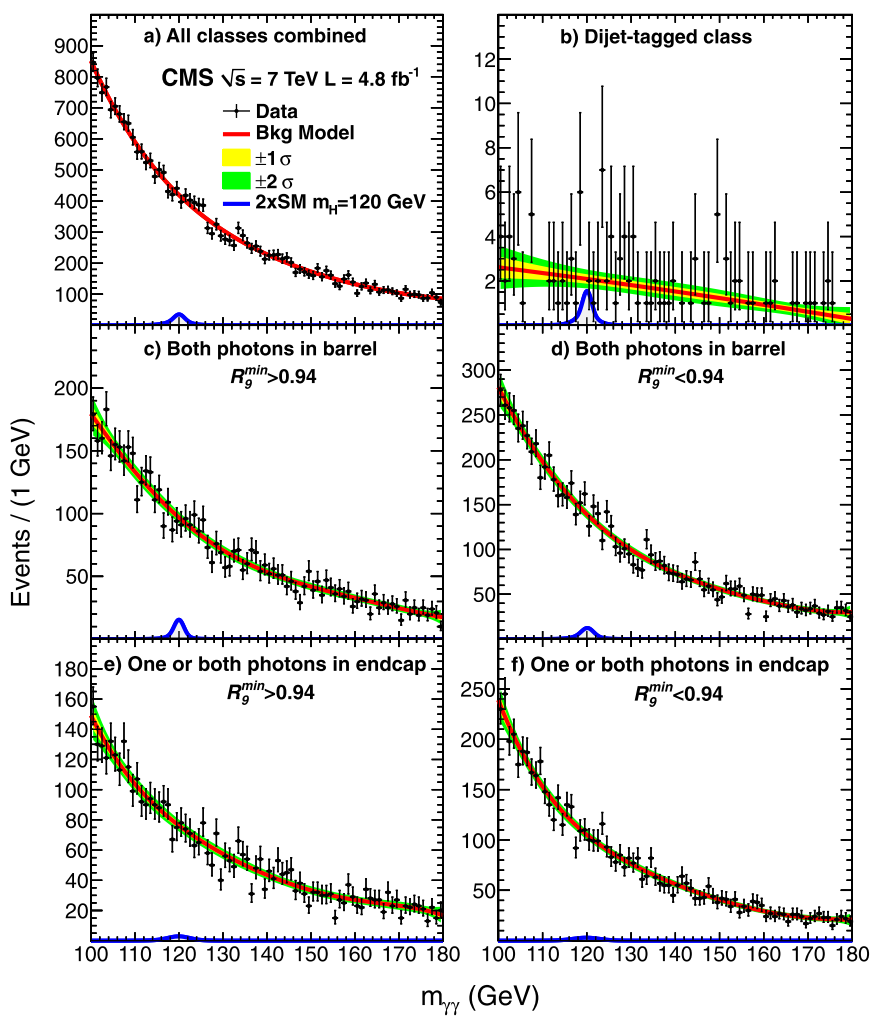

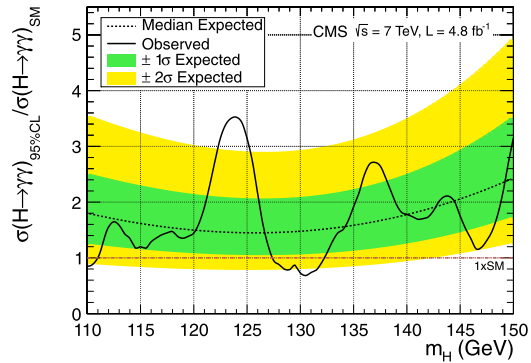

Figure 5. Background model fit to the $m_{y y}$ distribution for the five event classes, together with a simulated signal $\left(m_{\mathrm{H}}=120 \mathrm{GeV}\right)$. The magnitude of the simulated signal is what would be expected if its cross section were twice the SM expectation [4].

In the $\mathrm{WW}^{*}$ channel, the large sensitivity of this mode particularly at high masses has resulted in the elimination of most of the mass range for a SM Higgs boson. Figure 6 shows the distribution of transverse mass for the two cases, $\mathrm{H}$ to WW* to $(l v l v+0$-jets) and to $(l v l v+1$ jet) respectively. Figure 6 shows the ratio of measured limits to SM (without Higgs) expected rates from the ATLAS Collaboration [6] for combined $\mathrm{H}$ to $\mathrm{WW}^{*}$ to $l v l v$, for the complete mass range $110-600 \mathrm{GeV}$, and a detailed view in the low mass region. As can be seen, excellent sensitivity has already been achieved in this single channel from $125-300 \mathrm{GeV}$. CMS has published similar results in this channel [7]. 

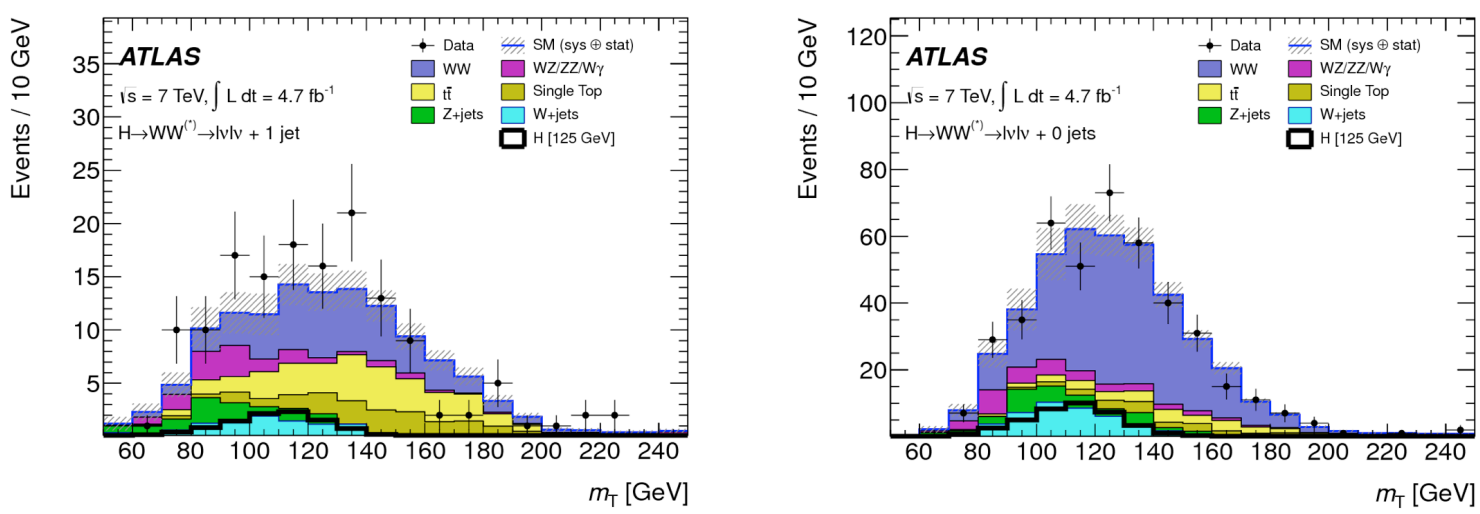

Figure 6. Transverse mass, $m_{\mathrm{T}}$, distribution in the 1-jet (left) and 0-jet (right) channels, in the low $m_{H}$ region. The expected signal for a $m_{H}=125 \mathrm{GeV}$ SM Higgs boson is shown. The hashed area shows the total uncertainty in the background [6].
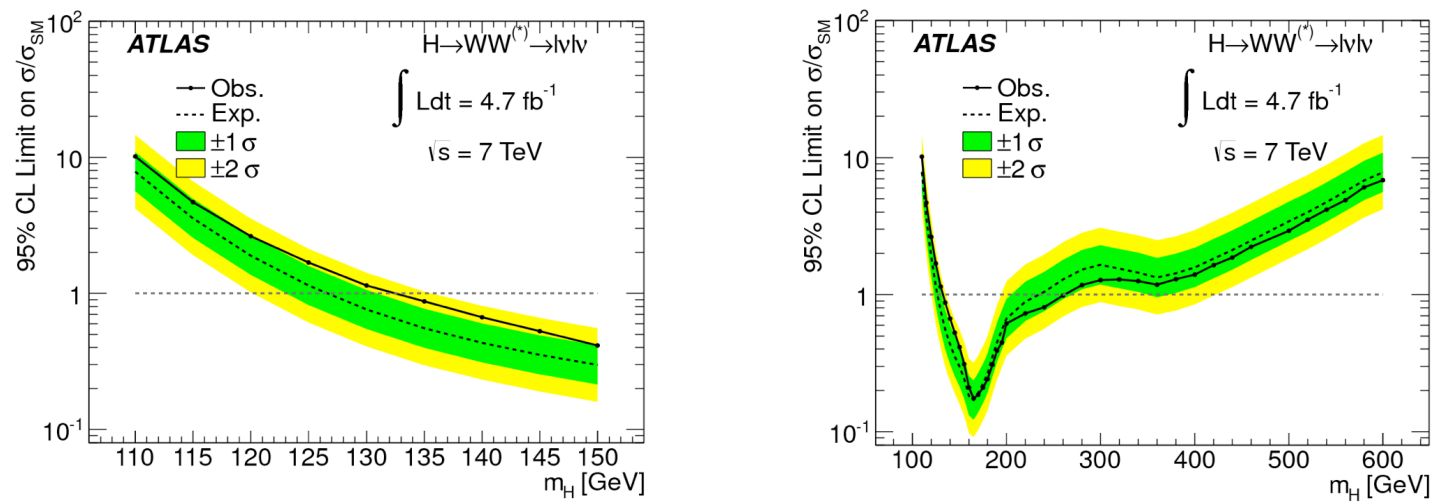

Figure 7. Observed (solid) and expected (dashed) upper limits on the Higgs boson production cross section, normalised to the SM cross section, for range $m_{H}<150 \mathrm{GeV}$ (left) and full mass range (right). The $\pm 1 \sigma$ and $\pm 2 \sigma$ uncertainty bands on the expected limit, are shown (see reference [6] for details). 

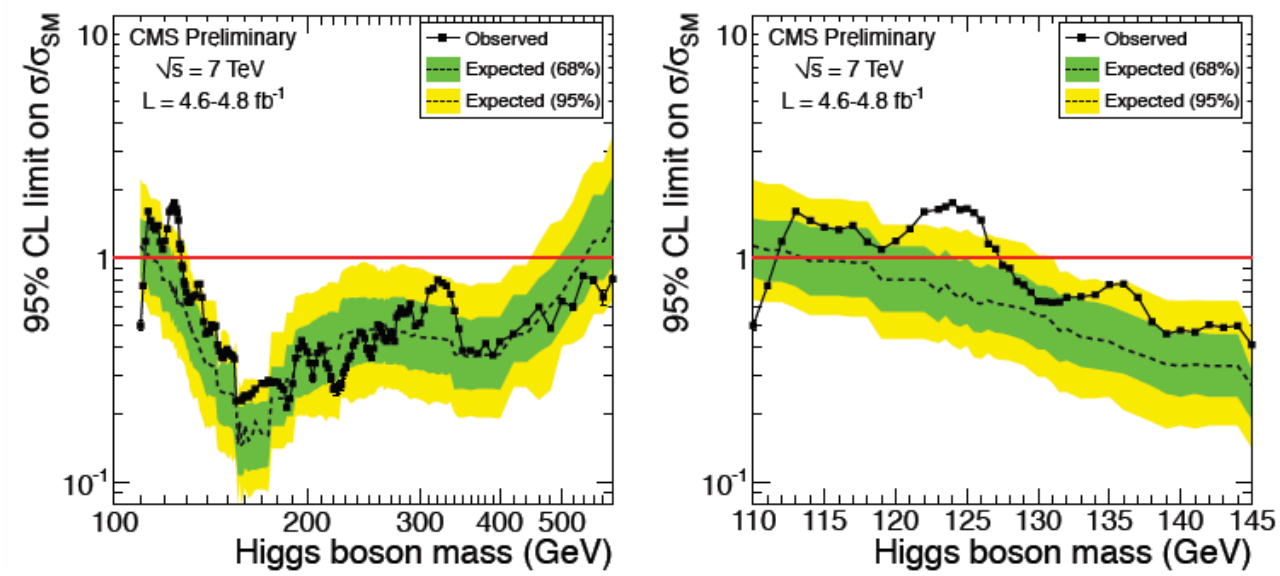

Figure 8 The CMS 95\% CL upper limits on ratio $\sigma / \sigma$ SM for the SM Higgs boson hypothesis as a function of $m_{H}$ in the range 110-600 GeV (left) and 110-145 GeV (right). The observed values are shown by the solid line, the dashed line indicates the expected median for background-only hypothesis, with bands indicating $\pm 1 \sigma$ and $\pm 2 \sigma$ uncertainty ranges. [8]
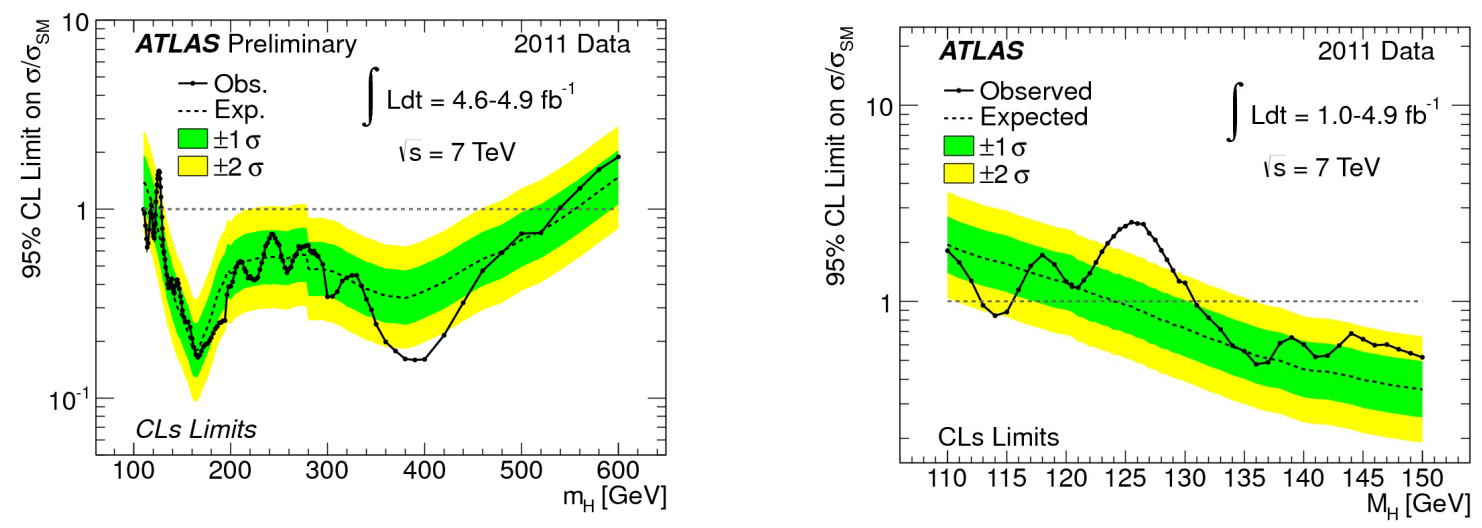

Figure 9. The combined ATLAS $95 \% \mathrm{CL}$ upper limits on the ratio $\sigma / \sigma \mathrm{SM}$ as a function of $\mathrm{m}_{\mathrm{H}}$; the solid curve indicates the observed limit and the dotted curve illustrates the median expected limit in the absence of a signal together with the $\pm 1 \sigma$ (green) and $\pm 2 \sigma$ (yellow) bands [9].

Combining the channels, both CMS [8] and ATLAS[9] have reported important limits for the existence of the SM Higgs boson over a broad range of masses. Figure 8(a) and (b) shows the ratio of measured cross section limit to expected SM cross sections (excluding the Higgs) over a broad mass region and a low mass region, respectively, from CMS. With the corresponding plots from ATLAS in Figures 9(a) and (b).

As can be seen much of the mass region has been excluded by these data. The exception to this is the low mass region where small excesses over the expected SM backgrounds have been observed. The excluded mass ranges for the two experiments are shown in table 1 . 


\begin{tabular}{|l|l|l|l|}
\hline Experiment & $\begin{array}{c}\text { Expected Sensitivity } \\
\text { SM Higgs Mass range }\end{array}$ & \multicolumn{2}{|c|}{ Excluded SM Higgs Mass Ranges } \\
\hline CMS & $114.5-543 \mathrm{GeV}$ & $127.5-600 \mathrm{GeV}$ & \\
\hline ATLAS & $120-555 \mathrm{GeV}$ & $\begin{array}{l}110-122.5 \mathrm{GeV} \\
(\operatorname{excl} 117.5-118.5)\end{array}$ & $129-539 \mathrm{GeV}$ \\
\hline
\end{tabular}

Conspicuous in these data is the tantalising excesses in the region $122.5-127.5 \mathrm{GeV}$ resulting in no exclusion of a potential SM Higgs boson in this mass range.

These limits are currently statistically limited, and have driven the LHC program for 2012, to achieve enough integrated luminosity to make a sensitive search in the $\sim 125 \mathrm{GeV}$ mass region.

Also absent is sensitivity to channels with the SM Higgs boson decaying into fermions, such as bb and tt. More data is awaited for these channels to be thoroughly explored in order to complete the basic search strategy for a SM Higgs.

\section{BSM}

\subsection{Kaluza-Klein resonances?}

Using a 2011 data sample corresponding to an integrated luminosity of $2.05 \mathrm{fb}^{-1}$ results from ATLAS shown in figure 10, offer no evidence for any resonance is in the top-antitop channel. In comparison with expectations for postulated narrow $Z$ ' bosons, the observed $95 \%$ CL limits range $9.3 \mathrm{pb}$ to $0.95 \mathrm{pb}$ were obtained for Z' masses in the range of $500-1300 \mathrm{GeV}$. The corresponding excluded mass region for a leptophobic topcolour $Z$ ' was $m_{Z},<880 \mathrm{GeV}$. The corresponding limit for Kaluza-Klein gluon excitations in the Randall-Sundrum model is $\mathrm{m}_{\mathrm{gKK}}<1130 \mathrm{GeV}$. [14]

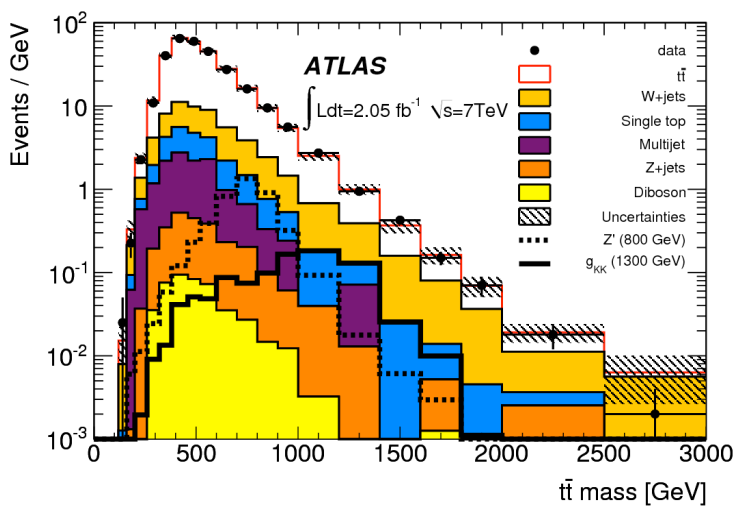

Figure 10. Reconstructed t-tbar mass in the (electron/muon) + jets channel, with the expectation from SM background and two signal masses, a $\mathrm{Z}$ ' boson with $\mathrm{m}_{\mathrm{Z}}$ ' $=800$ $\mathrm{GeV}$ and a KK gluon with $\mathrm{m}_{\mathrm{gKK}}=1300$ $\mathrm{GeV}$. Other backgrounds include single top, $\mathrm{Z}$ plus jets, diboson and multijet production. The hatched area shows the background normalization uncertainties

\subsection{Di-jet resonance search}

A search for di-jet resonances is a powerful tool for new physics discovery. Both ATLAS and CMS have published such searches. No resonances are observed. Upper limits at the $95 \%$ 
confidence level exclude excited quarks with mass less than $2.64 \mathrm{TeV}$ from $315 \mathrm{nb}^{-1}$ integrated luminosity and $2.49 \mathrm{TeV}$ from $1 \mathrm{fb}^{-1}$ (see figure 11).

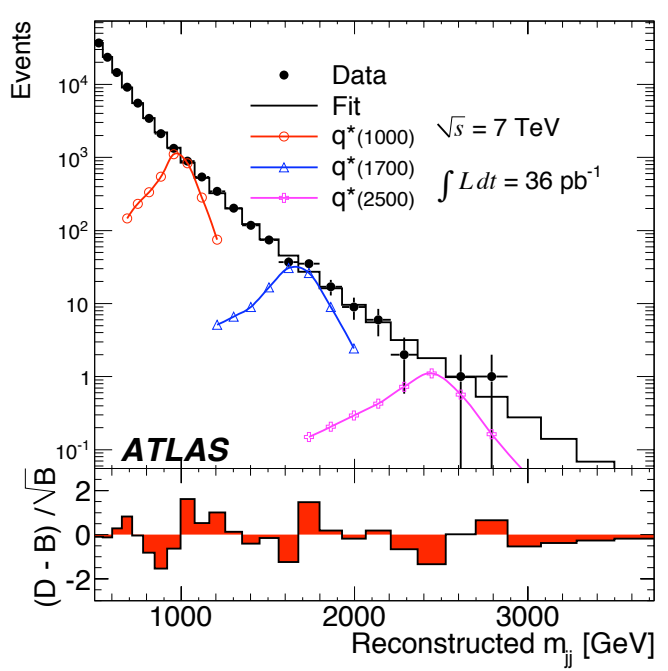

Figure 11. The measured dijet mass distribution (D - filled points) with fitted background distribution (B - histogram). The predicted $\mathrm{q}^{*}$ signals for excited-quark masses of 500,800 , and $1200 \mathrm{GeV}$ are overlaid [15]. (See also CMS results [16].)

\subsection{SUSY Constraints}

Using 2011 data with $\sim 5 \mathrm{fb}^{-1}$ a search for events with at jets and large missing transverse momentum. No excess above the expected backgrounds was observed. Within CMSSM with simplified models of gluino-gluino and squark-squark production, gluino masses below $1.0 \mathrm{TeV}$ and squark masses below $0.76 \mathrm{TeV}$ were excluded where the lightest supersymmetric particle mass is below $200 \mathrm{GeV}$ (CMS, figure 12). Gluino masses below $860 \mathrm{GeV}$ and squark masses below $1320 \mathrm{GeV}$ are excluded in simplified models containing only first two generation squarks, gluinos and a neutralino (ATLAS, figure 13). Changing the neutralino mass and allowing for a richer SUSY particle content, weakens the constraints (but gives more room to search further).

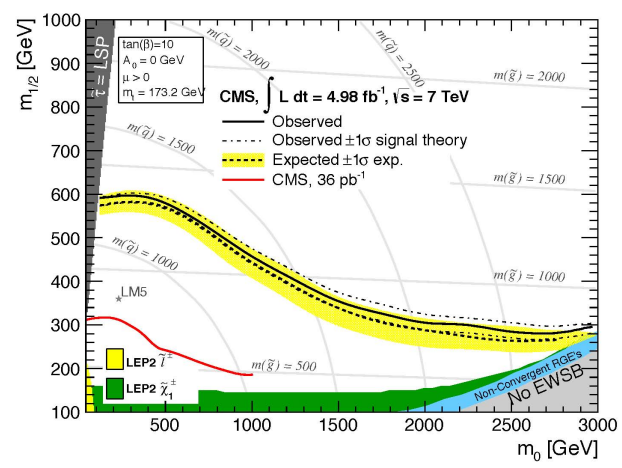

Figure 12. The observed and expected $95 \%$ C.L. limits in the CMSSM $\left(m_{0}, m_{1 / 2}\right)$ plane. The shaded region depicts the $\pm 1 \sigma$ expected limit. The dot-dashed curves show the observed band when the cross section is varied by theoretical uncertainties. The other CMSSM parameters are $\tan \beta=10$, $\mu>0$, and $A_{0}=0[11]$ 


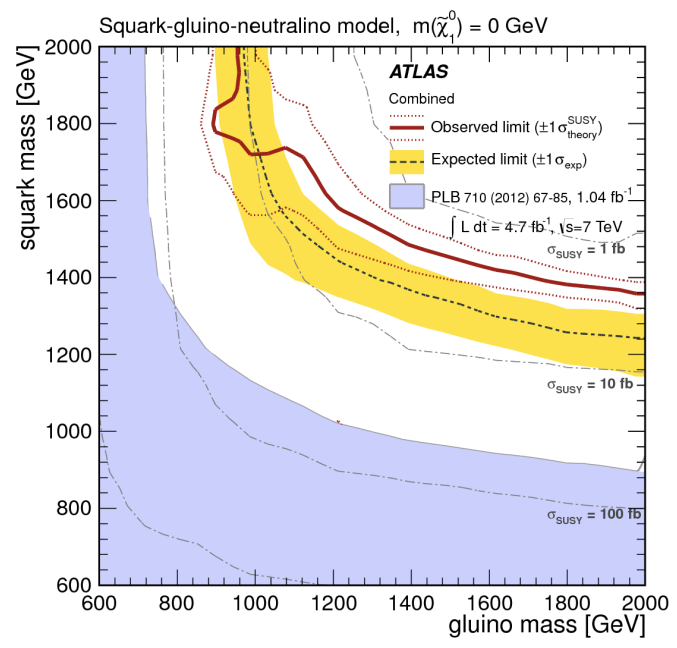

Figure 13. The 95\% CL exclusion limits on the $\left(m_{\text {gluino }}, m_{\text {squark }}\right)$-plane in a simplified MSSM scenario. Previous results from ATLAS are represented by the shaded region (blue). [10]

\subsection{Exotics Searches}

Many channels are being searched for evidence of physics beyond the standard model, by both the ATLAS and CMS collaborations. A (not complete) tabulation of such exotic channels is present for ATLAS data in figure 14 [12]. CMS provides a similar exclusion summary [13]. This plot gives an idea of the mass limits allowed for various channels. The shaded bars in each of the rows (corresponding to different channels) indicate the excluded mass range for the particles decaying to the indicated final states.

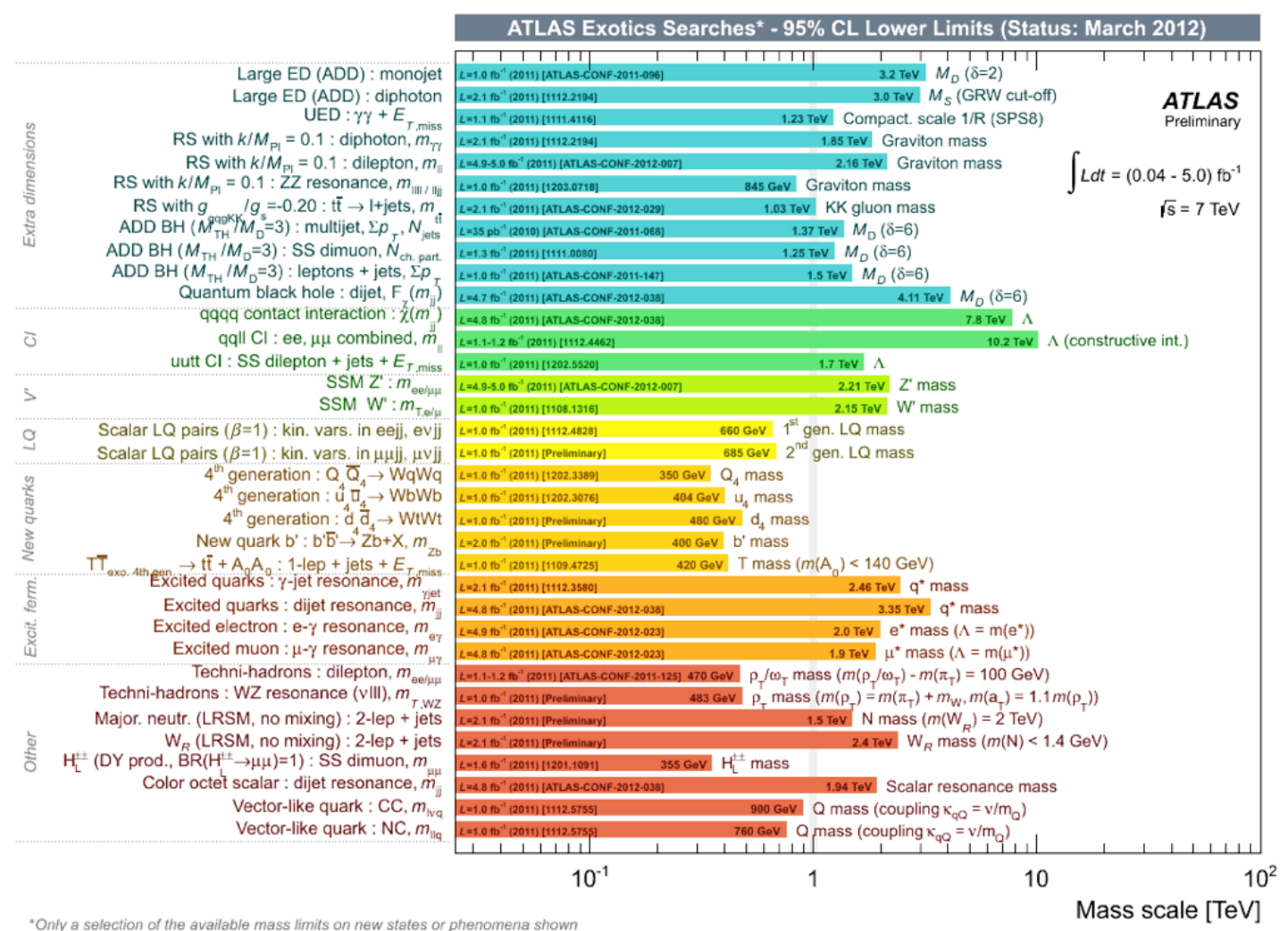

Figure 14. Summary of mass limits for various "exotic channels" form the ATLAS collaboration [12] 


\section{B-Physics and the SM}

The flavour-changing neutral current decays, such as $B^{0} \rightarrow K^{0} \mu^{+} \mu^{-}$are forbidden at tree level in the Standard Model. Ratios where the leading form factor uncertainties cancel can be powerful tools to search for new physics. One such ration is he CP averaged isospin asymmetry defined as

$$
A_{I}=\frac{\Gamma\left(B^{0} \rightarrow K^{0} \mu^{+} \mu^{-}\right)-\Gamma\left(B^{+} \rightarrow K^{+} \mu^{+} \mu^{-}\right)}{\Gamma\left(B^{0} \rightarrow K^{0} \mu^{+} \mu^{-}\right)+\Gamma\left(B^{+} \rightarrow K^{+} \mu^{+} \mu^{-}\right)}
$$

There is no precise prediction for AI but it is also expected to be close to zero in the SM. However, LHCb (see figure 15) finds a small but significant asymmetry, with significance of $4.4 \sigma$ from zero integrated across $\mathrm{q}^{2}[17]$.

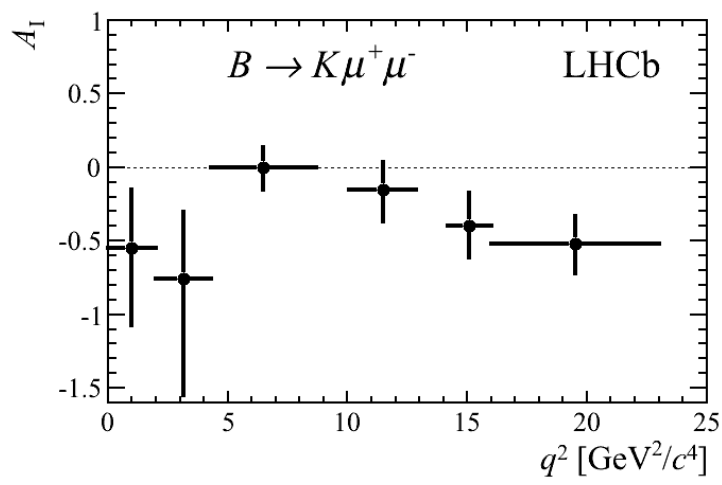

Figure 15. Isospin asymmetry of $B^{0} \rightarrow K^{0} \mu^{+} \mu^{-}[17]$

\section{Pb-Pb Collisions}

Azimuthal distribution of particles in the plane perpendicular to the beam direction is called the azimuthal anisotropy and is usually characterized by the Fourier coefficients:

$$
v_{n}=\left\langle\cos \left[n\left(\phi-\Psi_{n}\right)\right]\right\rangle
$$

where $\phi$ is the azimuthal angle of the particle, $\Psi_{n}$ is the angle of the initial state spatial plane of symmetry, and $\mathrm{n}$ is the order of the harmonic. Because the planes of symmetry $\Psi_{n}$ are not known experimentally, the anisotropic flow coefficients are estimated from measured correlations between the observed particles. The second Fourier coefficient $\mathrm{v}_{2}$ is called elliptic flow.

First measurements of particle flow v3, v4 and v5 have been shown by ALICE in $\mathrm{Pb}-\mathrm{Pb}$ collisions at cms energy $2.76 \mathrm{TeV}$, and are consistent with hydrodynamic models of particle flow in high- $\mathrm{p}_{\mathrm{T}}$ interactions (see figure 16, [18]). 


\section{Future Plans}

The clear aim of CERN and the LHC, ATLAS and CMS teams is to provide enough data to be sensitive to a SM Higgs discovery in 2012, before the scheduled long shutdown from 2013. The data needed at $8 \mathrm{TeV}$ in the $\mathrm{cms}$, is calculated to be an integrated luminosity of $11.5 \mathrm{fb}^{-1}$. Current estimations indicate that this aim is well within reach.

Two other aims before the shutdown include machine studies at $25 \mathrm{~ns}$ bunch spacing, followed by a run with $\mathrm{p}-\mathrm{Pb}$ collisions.

Current operation has the bunch spacing at 50ns, resulting in a large mean interaction rate per bunch crossing, as indicated above. For further increases in the luminosity and for running at higher energy, the $40 \mathrm{MHz}$ operation is essential.

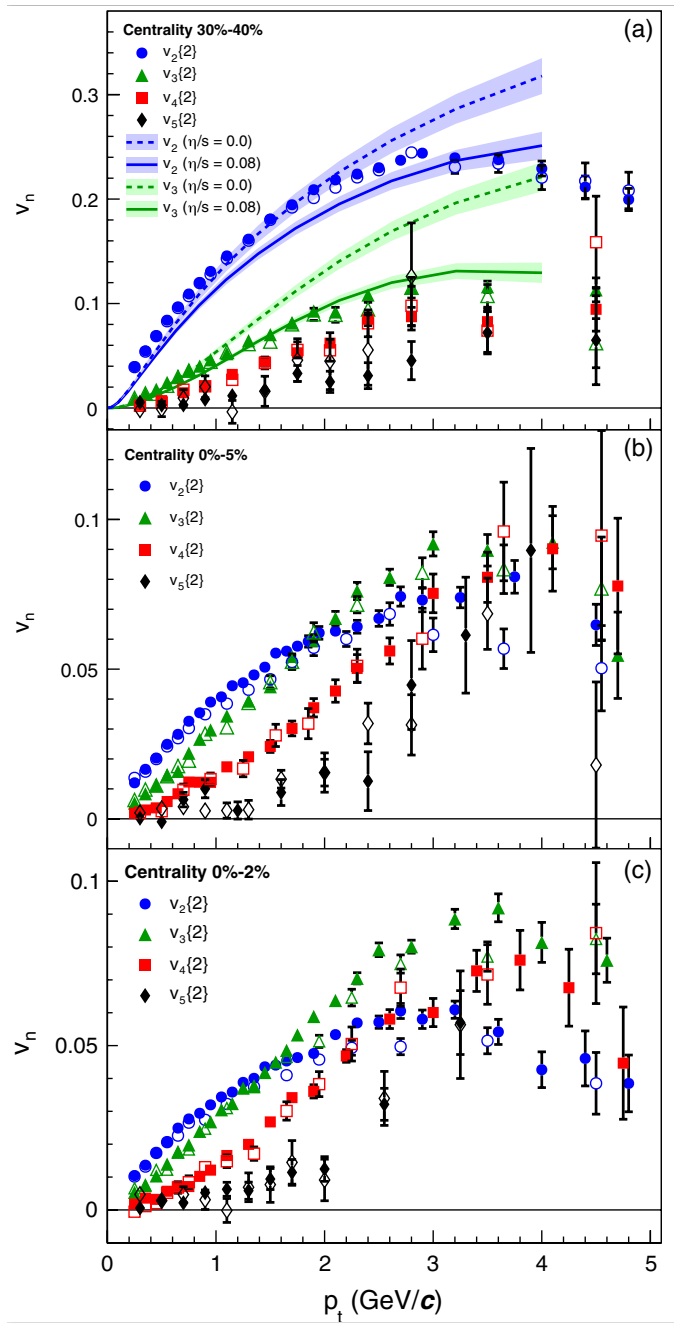

Figure 16. $v_{2}, v_{3}, v_{4}, v_{5}$ as a function of transverse momentum and for three event centralities. The full and open symbols are for $\Delta \eta>0.2$ and $\Delta \eta>1.0$, respectively. (a) $30 \%-40 \%$ compared to hydrodynamic model calculations, (b) $0 \%-5 \%$ centrality percentile, (c) $0 \%-2 \%$ centrality percentile. [18] 


\section{Summary}

The LHC and its experiments have had a very successful initial operation. The SM has been "rediscovered", much of the potential mass range for the SM Higgs boson has already been eliminated and tantalising hints of a low mass Higgs have been reported. Searches for evidence of supersymmetry have pushed the mass scale of the simplest SUSY models into the $\mathrm{TeV}$ region, and exotics mass scales are similarly well into the TeV region. LHCb has made high precision measurements of many rare b-decay branching ratios, and has uncovered an unexplained isospin asymmetry in $B \rightarrow K \mu^{+} \mu^{-}$. Measurement of particle flow and effects of quark-gluon plasma observed in $\mathrm{Pb}-\mathrm{Pb}$ collisions have been reported.

In the words of the CERN Director General Professor Rolf Heuer (Email to CERN Users, June 2012), “ . . a at less than two weeks from the start of the ICHEP conference, the news from the experiments is exciting. As I reported to Council, the hints that were reported in the 2011 data last December are still present in the 2012 data. Furthermore, refinements of the analysis of the 2011 data have confirmed the hints reported in December. It is too early for the experiments to say whether the significance is enough to claim a discovery, but whatever the news, it will be reported at CERN on Wednesday 4 July with a live two-way video link to the scientists gathering in Melbourne for ICHEP2012."

The news is good, and the expectations are high for important updates on the Higgs search as early as the ICHEP2012 conference in two weeks time.

\section{References}

[1] https://twiki.cern.ch/twiki/bin/view/AtlasPublic/LuminosityPublicResults\#Multiple_Year_Collis ion_Plots (and references therein)

[2] https://twiki.cern.ch/twiki/bin/view/AtlasPublic/CombinedSummaryPlots

[3] https://twiki.cern.ch/twiki/bin/view/CMSPublic/LumiPublicResults\#Multi_year_Collisions_Plots

[4] S. Chatrchyan, et al., (CMS Collaboration) Phys. Lett. B 710 (2012) 403-425

[5] G. Aad, et al., (ATLAS Collaboration) Phys. Rev. Lett.108 (2012) 111803

[6] G. Aad, et al., (ATLAS Collaboration) Phys. Lett. B 716, (2012) 62-81

[7] S. Chatrchyan, et al., (CMS Collaboration) Physics Letters B 710 (2012) 91-113

[8] S. Chatrchyan, et al., (CMS Collaboration) Physics Letters B 710 (2012) 26-48

[9] G. Aad, et al., (ATLAS Collaboration) Physics Letters B 710 (2012) 49-66

[10] G. Aad, et al. (ATLAS Collaboration) arXiv:1208.0949v2 [hep-ex]

[11] S. Chatrchyan et al. (CMS Collaboration) Phys. Rev. Lett. 109 (2012) 171803

[12]For recently upgraded ATLAS exotics search results see: https://twiki.cern.ch/twiki/pub/AtlasPublic/CombinedSummaryPlots/AtlasSearches_exotics_hcp12. pdf.) 
[13]https://twiki.cern.ch/twiki/bin/view/CMSPublic/PhysicsResultsEXO\#CMS_EXO_Summary_of_ Mass_Limits

[14]e-Print: arXiv:1205.5371 [hep-ex]

[15]G. Aad et al. (ATLAS Collaboration), Phys. Rev .Lett. 105 (2010) 161801

[16]S. Chatrchyan et al. (CMS Collaboration) Phys. Lett. B 704 (2011) 123-142

[17]Aaij, R. et al. (LHCb Collaboration) JHEP 07 (2012) 133

[18]K. Aamodt et al. (ALICE Collaboration) Phys. Rev. Lett. 107, 032301 (2011) 